\title{
Misurazione della spesa energetica mediante la camera metabolica nello studio dei fenotipi dell'obesità
}

\author{
Alessio Basolo ${ }^{1} \cdot$ Paola Fierabracci $^{1} \cdot$ Ferruccio Santini $^{1}$
}

Accettato: 23 settembre 2021 / Pubblicato online: 12 gennaio 2022

(c) The Author(s) 2022

\section{Sommario}

La capacità di modulare l'introito calorico in risposta ai cambiamenti della richiesta energetica è essenziale per la sopravvivenza dell'individuo. L'apparente spontaneità con cui decidiamo di alimentarci dipende da una complessa interazione tra percezioni visive olfattive e cognitive e il sistema nervoso centrale che integra a livello ipotalamico i segnali periferici relativi allo stato nutrizionale. La conservazione dell' equilibrio energetico può essere considerata un processo dinamico e, sotto controllo fisiologico ideale, le variazioni di un componente (spesa energetica) provocano cambiamenti compensatori biologici e/o comportamentali nell'altra parte del sistema (introito calorico) e viceversa. Nella vita di tutti i giorni un abbinamento così perfetto tra apporto energetico e dispendio energetico è difficilmente raggiungibile e il tessuto adiposo funge da deposito dinamico, proteggendo dalle inevitabili deviazioni dell'equazione di equilibrio. Recenti studi hanno dimostrato che la risposta adattativa della spesa energetica a differenti interventi dietetici (alimentazione eccessiva o restrizione calorica) identifica la presenza di due differenti fenotipi metabolici ("dissipatore" e "risparmiatore"). In questa rassegna verranno discussi i principi fondamentali dell'equazione del bilancio energetico e il loro metodo di misurazione mediante camera metabolica. Verranno inoltre descritti i due diversi fenotipi metabolici che possono indicare la propensione di un individuo a essere più $o$ meno incline allo sviluppo dell'obesità.

Parole chiave Spesa energetica $\cdot$ Termogenesi adattativa $\cdot$ Regolazione del peso corporeo $\cdot$ Fenotipi metabolici

\section{Introduzione}

L'obesità è un problema di salute pubblica in continua crescita e, su scala mondiale, il numero dei soggetti con obesità è all'incirca triplicato dal 1975 ad oggi [1]. Un'alterazione dell'equilibrio tra assunzione di cibo (EI) e dispendio energetico (EE) concorre alla patogenesi dell'obesità. Nonostante la soluzione al "problema" dell'obesità possa apparire facilmente raggiungibile aumentando il dispendio calorico e riducendo l'assunzione di energia, il fallimento dei tentativi di cambiare lo stile di vita in un ambiente obesogenico indica che la nostra comprensione delle complesse interazioni tra genetica, fattori ambientali e relazioni sociali è ancora

Proposto da P. Fierabracci e F. Santini.

A. Basolo

alessio.basolo@med.unipi.it

1 Sezione Centro Obesità e Lipodistrofie, Unità operativa di Endocrinologia 1, Dipartimento di Medicina Clinica e Sperimentale, Azienda Ospedaliero Universitaria Pisana, Pisa, Italia insufficiente per correggere efficacemente e in modo durevole il bilancio energetico nella maggior parte delle persone. Una delle principali sfide nella ricerca sull'obesità è la misurazione accurata dell' apporto calorico e del dispendio energetico dell'individuo, al fine di identificare le associazioni causali tra i componenti dell'omeostasi energetica e di definire con precisione le variazioni del bilancio energetico in risposta a diversi stimoli. La precisa misurazione del dispendio energetico è difficile nella vita di ogni giorno ma è resa possibile utilizzando un ambiente controllato come la camera metabolica [2], un sistema di calorimetria indiretta che consente di analizzare gli scambi gassosi dell'individuo per periodi di tempo prolungati. Attraverso le misurazioni della risposta adattativa della $\mathrm{EE}$ a interventi dietetici differenti (alimentazione eccessiva o una restrizione calorica) sono stati identificati 2 differenti fenotipi metabolici ("dissipatore" e "risparmiatore"). In questa rassegna verranno discussi i principi fondamentali dell'equazione del bilancio energetico e il loro metodo di misurazione mediante camera metabolica. Verranno inoltre descritti i due diversi fenotipi metabolici 
Fig. 1 Bilancio energetico e tessuto adiposo. Lo squilibrio nella regolazione del bilancio energetico può essere causato da diversi fattori come disponibilità alimentari, composizione in macronutrienti, attività fisica, ritmi circadiani, temperatura ambientale e altre sostanze esogene. Il tessuto adiposo svolge un ruolo attivo nella regolazione del bilancio energetico, non solo come sito per l'immagazzinamento dei lipidi, ma anche essendo coinvolto nella generazione di calore (nel tessuto adiposo bruno) e nella secrezione di adipochine. L'organo adiposo funge da deposito energetico dinamico che trasmette costantemente segnali al sistema nervoso centrale per comunicare la quantità di riserve di energia e suscitare risposte compensatorie
Disponibilità alimentari, composizione in macronutrienti, attività fisica, ritmi circadiani, temperatura ambientale, altre sostanze esogene

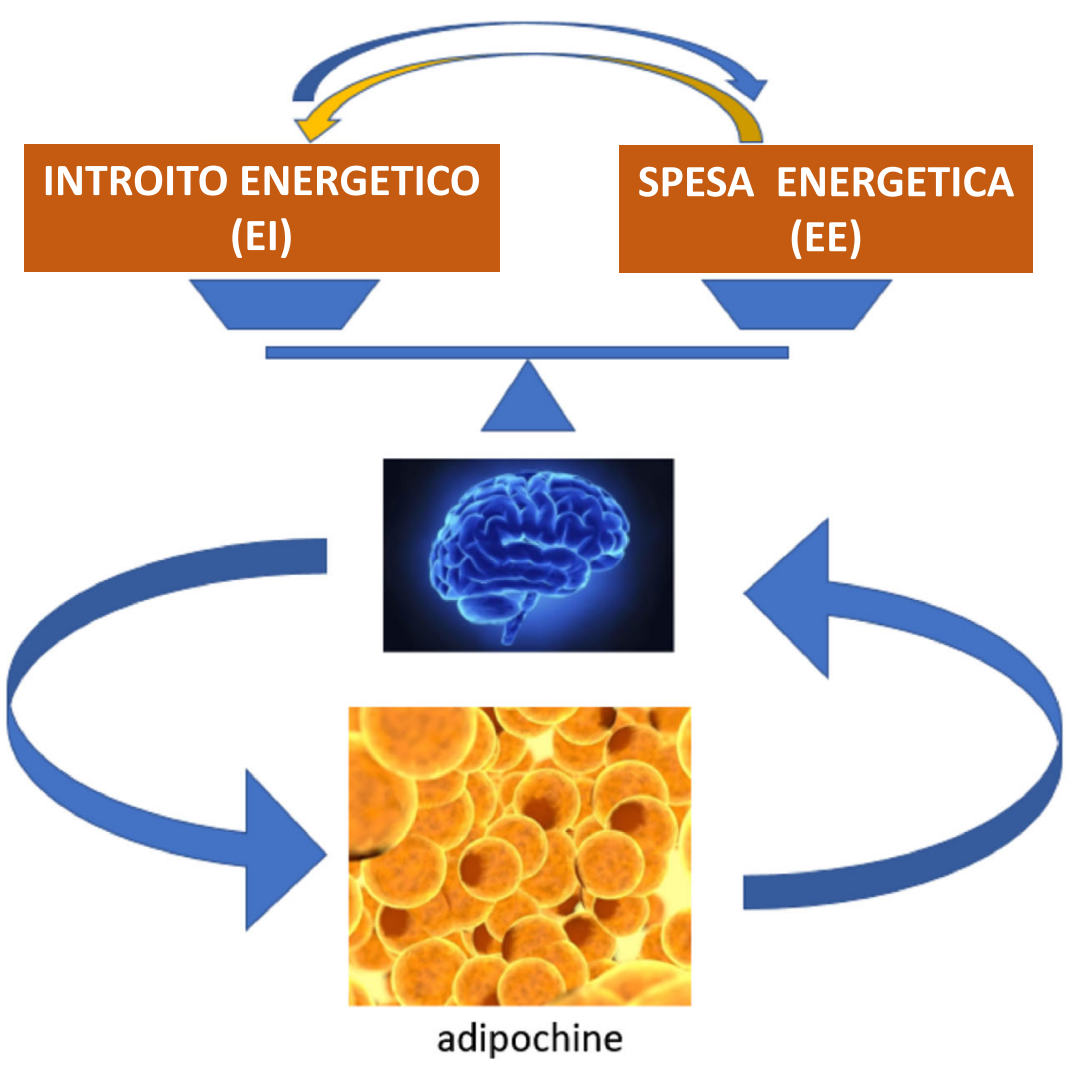

che possono indicare la propensione di un individuo a essere più o meno incline allo sviluppo dell'obesità.

\section{Il controllo centrale dell'introito calorico e del peso corporeo}

La capacità di modulare l'introito calorico in risposta ai cambiamenti della richiesta energetica è essenziale per la sopravvivenza dell'individuo. L'apparente spontaneità con cui decidiamo di alimentarci dipende da una complessa interazione tra percezioni visive, olfattive e cognitive e il sistema nervoso centrale che integra a livello ipotalamico i segnali periferici relativi allo stato nutrizionale. Questa integrazione, a seguito dell'ingestione di cibo, comporta l'induzione da parte dell'ipotalamo di segnali di sazietà. Un altro fattore che condiziona l'assunzione di cibo è l'entità delle riserve energetiche dell'organismo sotto forma di tessuto adiposo. In questo modo, attraverso numerosi mediatori endocrini e neuronali, le informazioni riguardanti lo stato nutrizionale e i depositi energetici vengono comunicate al cervello, dove vengono integrate con afferenze psico-sensoriali per modulare l'impulso a introdurre ulteriore cibo [3]. Interferenze derivanti da numerosi fattori ambientali possono intervenire nel controllo dell'equazione energetica (Fig. 1).

\section{Il significato del bilancio energetico: da una prospettiva statica a una dinamica}

La patogenesi del sovrappeso e dell'obesità viene generalmente spiegata in base a un semplice modello statico per il quale un bilancio energetico cronicamente positivo (apporto calorico superiore al dispendio) porta all'aumento del peso corporeo e allo sviluppo del sovrappeso/obesità, dovuto all'immagazzinamento del surplus sotto forma di grasso corporeo [4]. Questo modello presuppone che le variazioni nell' apporto calorico e nell' attività fisica modifichino indipendentemente le componenti del bilancio energetico. In realtà, l'assunzione di energia e il dispendio energetico dipendono l'uno dall'altro e sono regolati da un modello più complesso, che mira a mantenere il peso corporeo e le riserve di energia del corpo entro un intervallo definito. Quindi, la conservazione dell'equilibrio energetico può essere considerata un processo dinamico e, sotto controllo fisiologico ideale, le variazioni di un componente (spesa energetica) provocano cambiamenti compensatori biologici e/o comportamentali nell'altra parte del sistema (introito calorico) e viceversa. Secondo questa teoria, una riduzione dell'apporto calorico induce una corrispondente diminuzione del dispendio energetico, mentre un aumento dell'attività fisica è seguito dalla stimolazione della fame per aumentare l'apporto 
Fig. 2 Camera metabolica. La camera metabolica è una comune stanza (b) paragonabile a un ambiente domestico, in cui i flussi d'aria in entrata (aria medicale introdotta) e in uscita (aria della stanza + aria espirata) sono controllati da strumentazioni ad alta precisione (controllori digitali di flusso) in grado di misurare in tempo reale il consumo di ossigeno $\left(\mathrm{VO}_{2}\right)$ e la produzione di anidride carbonica $\left(\mathrm{VCO}_{2}\right)(\mathbf{a})$
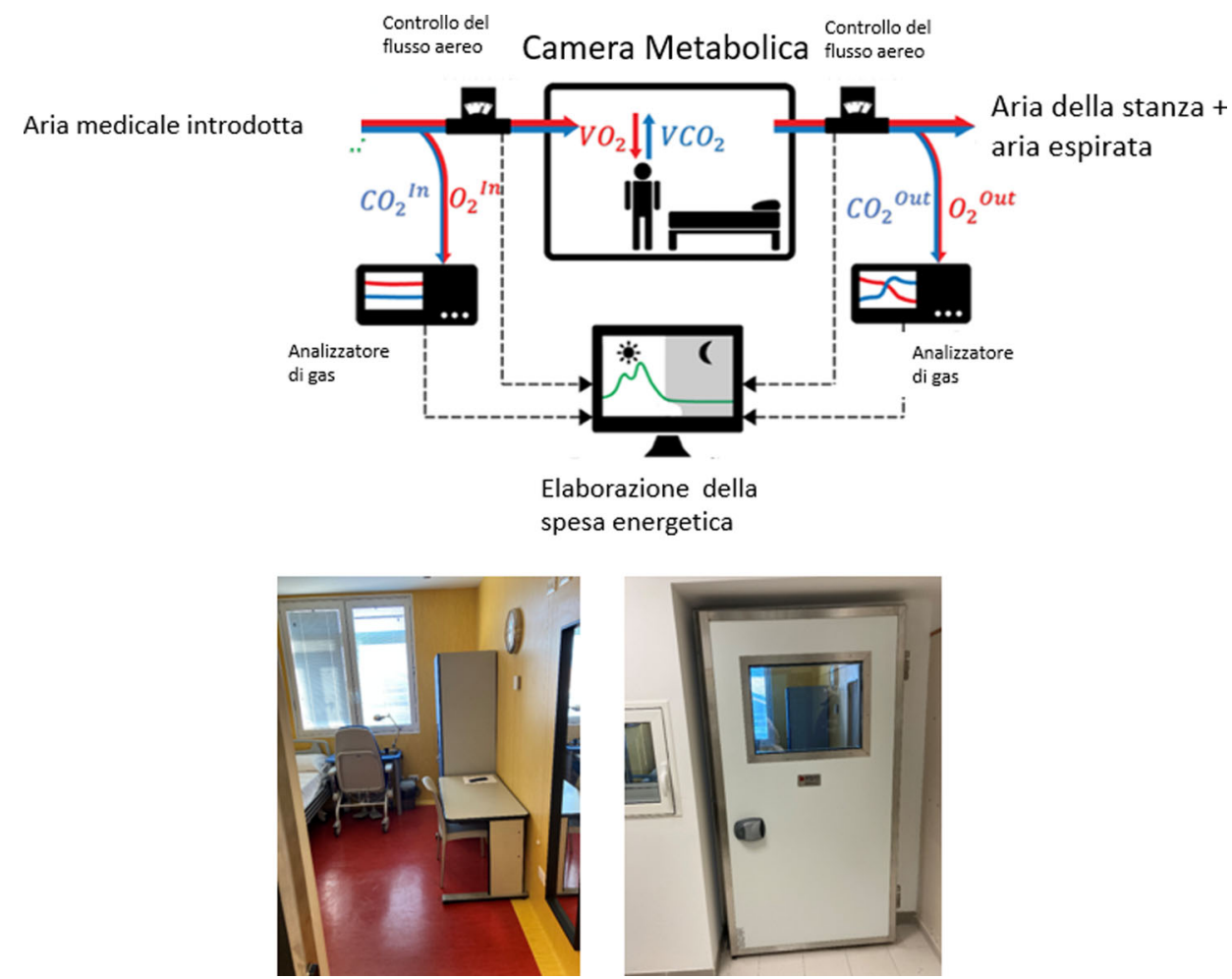

calorico. Nella vita di tutti i giorni un abbinamento così perfetto tra apporto energetico e dispendio energetico è difficilmente raggiungibile e il tessuto adiposo funge da deposito dinamico, proteggendo dalle inevitabili deviazioni dell'equazione di equilibrio e trasmettendo costantemente segnali al sistema nervoso centrale attraverso la via della leptina per comunicare la quantità di riserve (Fig. 1).

\section{Misurazione e regolazione del bilancio energetico: la camera metabolica}

La spesa energetica dell'organismo (EE) può essere misurata in modo accurato e continuo all'interno della camera metabolica, un calorimetro indiretto a circuito aperto [5]. La camera metabolica è una comune stanza paragonabile a un ambiente domestico, in cui i flussi d'aria in entrata e in uscita sono controllati da strumentazioni ad alta precisione (controllori digitali di flusso) in grado di misurare in tempo reale il consumo di ossigeno $\left(\mathrm{VO}_{2}\right)$ e la produzione di anidride carbonica $\left(\mathrm{VCO}_{2}\right)$ (Fig. 2). Misurando il $\mathrm{VO}_{2}$ e la $\mathrm{VCO}_{2}$, la camera metabolica fornisce una stima precisa dell'energia consumata da un soggetto umano ogni minuto nell' arco delle 24 ore. Questo metodo è considerato il gold standard per la misura del dispendio energetico grazie alla capacità di distinguere le componenti giornaliere del dispendio energetico come la spesa energetica a riposo (resting metabolic rate, RMR), l'effetto termico del cibo (thermic effect offood,
TEF) e il costo energetico dell' attività fisica (physical activity, PA). Inoltre, misurando l'azoto nelle urine delle 24 ore viene calcolata la proporzione in cui avviene l'ossidazione dei singoli substrati energetici (carboidrati, lipidi e proteine). I componenti e la distribuzione relativa del consumo energetico giornaliero sono illustrati nella Fig. 3.

In condizioni di vita sedentaria, la RMR è la componente principale dell'EE delle $24 \mathrm{~h}(60-70 \%)$ e può essere ulteriormente classificata nell'energia spesa durante il sonno (sleeping metabolic rate, SMR) e nell'energia spesa per rimanere svegli (cost of arousal, CoA), escludendo l'energia derivante dall'attività fisica [6]. Il principale determinante della RMR è la massa magra che rende conto di circa il $70 \%$ della varianza [7], mentre la massa grassa, il sesso, l'età, le etnie e i tratti familiari spiegano un ulteriore $15 \%$ circa [8]. La spesa energetica derivante dell'attività fisica (PA), definita come l'energia consumata durante l'esercizio spontaneo e volontario, è la componente più soggetta a variazioni, rappresentando $\sim 15 \%$ dell'EE in soggetti molto sedentari e arrivando a $\sim 50 \%$ in soggetti molto attivi [9]. Analogamente alla RMR, il costo energetico della PA dipende dalla composizione corporea, dall' età, dal sesso, dai tratti genetici e da diversi stimoli ambientali. Il TEF, definito come l'aumento di energia necessaria per digerire, assorbire, assimilare e immagazzinare i nutrienti dopo l'ingestione del pasto, rappresenta $\sim 10 \%$ dell'EE delle 24 ore, quando i soggetti si trovano in stato di equilibrio energetico con una dieta occidentale [10], seppure con una grande variabilità interin- 
Fig. 3 Componenti del consumo energetico giornaliero

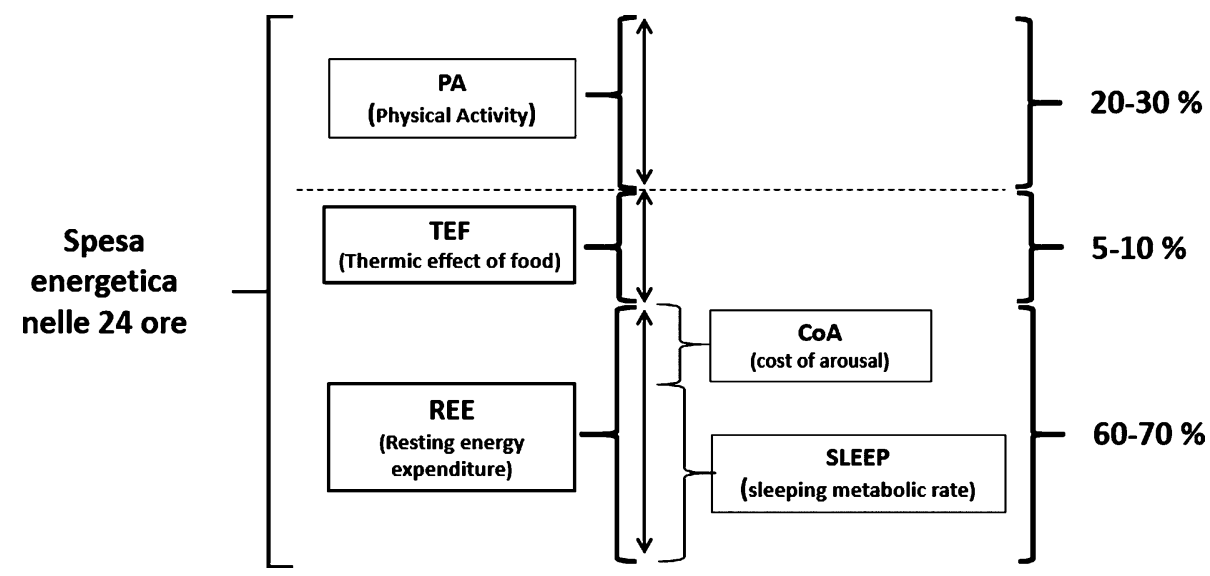

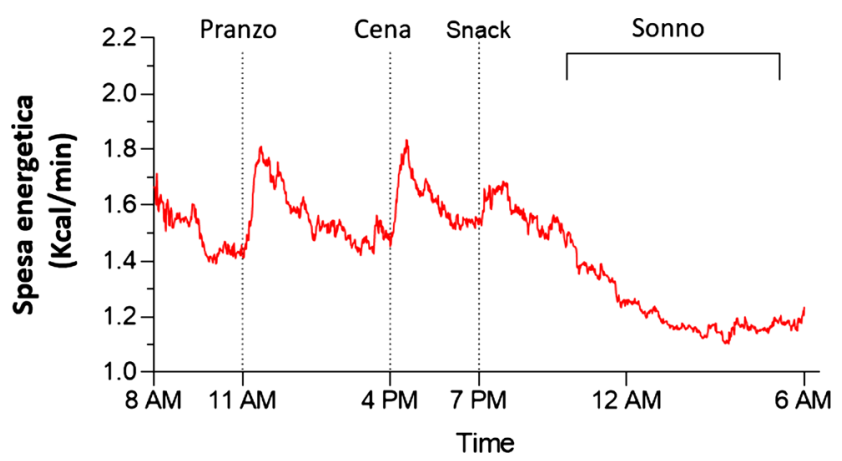

Fig. 4 Esempio di un tracciato della spesa energetica di un individuo nelle 24 ore misurato con la camera metabolica

dividuale. I componenti del TEF sono il suo costo obbligatorio, ovvero l'energia utilizzata per metabolizzare i nutrienti ingeriti, e la sua componente facoltativa che riflette la risposta individuale all'eccesso o alla restrizione dell' apporto alimentare. Il costo obbligatorio dipende dalla quantità e dal tipo di macronutrienti ingeriti, pari rispettivamente al 5-10\%, 20-30\% e 0-3\% delle calorie introdotte con carboidrati, proteine e grassi, rispettivamente [11]. È stato inoltre ipotizzato che l'obesità possa ridurre il TEF durante le condizioni di riposo, esercizio fisico e post-esercizio [12]. Nella Fig. 4 è riportato un esempio di un tracciato della spesa energetica delle 24 ore di un individuo, misurato tramite la camera calorimetrica, che illustra le variazioni della spesa energetica in condizioni di sonno, veglia e dopo i pasti.

Da un punto di vista evolutivo, l'interdipendenza tra fabbisogno energetico e apporto calorico appare finalizzata al mantenimento dell'equilibrio energetico, necessario per consentire la sopravvivenza e la riproduzione in contesti di vita variabili. Studi recenti hanno ipotizzato l'esistenza di un "sensore di energia", in grado di trasmettere al sistema nervoso centrale segnali relativi alla spesa energetica, che intervengono sul senso di fame per adeguare l'apporto calorico in base alle necessità dell'individuo. A sostegno di questa ipotesi, è stato dimostrato che l'EE a riposo è positivamen- te associata alla sensazione di fame e all'apporto energetico giornaliero in condizioni di alimentazione libera [13]. La massa magra, la parte metabolicamente più attiva del corpo umano, è il principale determinante dell'EE [2], per cui è stato ipotizzato che potrebbe intervenire come mediatore dei segnali inviati al sistema nervoso centrale dal "sensore di energia”. In realtà, è stato recentemente dimostrato che l'EE è associata all'assunzione di introito calorico indipendentemente dalla FFM, a indicare l'esistenza di altri meccanismi responsabili di tale mediazione [13].

La completa efficienza di questo meccanismo di feedback è messa in dubbio dal fatto che la maggior parte delle persone, sotto la pressione di un ambiente obesogenico, aumenta le proprie riserve di grasso corporeo oltre ciò che è considerato fisiologico. Gli studi condotti a tale proposito sono numerosi e non sempre di facile interpretazione. In uno dei primi studi, i soggetti con valori più bassi di RMR ed EE nelle 24 ore andavano incontro a un aumento del peso corporeo [14], facendo ipotizzare un apporto alimentare squilibrato in eccesso pur in presenza di bassi consumi, come espressione di una tendenza costitutiva al mantenimento di un bilancio energetico positivo e all'aumento delle riserve di energia. Al contrario, in uno studio condotto in individui nigeriani magri, l'aumento di peso si riscontrava nei soggetti con EE più elevata [15], indicando la predisposizione a ipercompensare l'aumento delle richieste energetiche con un maggiore introito alimentare. Questi risultati apparentemente contrastanti possono essere ricondotti a un' unica spiegazione, considerando che la misurazione dell'EE deve essere sempre valutata relativamente all'introito calorico. In effetti, quando vengono misurate entrambe le componenti dell'equazione, è il rapporto tra introito calorico ed EE che predice l'andamento del peso corporeo: in una popolazione di Nativi Americani sovrappeso, un'assunzione di cibo relativamente maggiore rispetto all'EE nelle 24 ore prediceva un aumento di peso a 2 anni di follow-up [16]. 


\section{Fenotipi metabolici: risparmiatore e dissipatore di energia}

L'ipotesi del fenotipo "risparmiatore" comprende l'idea ancestrale che fosse vantaggioso conservare energia per il mantenimento del peso corporeo nelle popolazioni di cacciatori/raccoglitori in epoca preistorica. Nell'ambiente obesogenico odierno, il risparmio energetico non sarebbe più vantaggioso in quanto porta a un eccessivo accumulo di riserve che non vengono utilizzate. Nonostante in linea generale questo modello fornisca una solida base teorica per spiegare la patogenesi dell'obesità, non può essere considerato valido in termini assoluti poiché non rende conto della resistenza di alcuni individui a sviluppare obesità anche in ambienti particolarmente favorenti. In uno studio condotto su 14 uomini, l'EE nelle 24 ore diminuiva di circa il $10 \%$ rispetto al valore basale durante il digiuno di 48 ore, mentre aumentava di circa il $10 \%$ a seguito di sovralimentazione [17]. Veniva inoltre osservato che i soggetti con una minor riduzione dell'EE in risposta al digiuno tendevano ad avere un maggior aumento dell' $E E$ in risposta all'iperalimentazione e viceversa. Questa osservazione iniziale è stata successivamente confermata in condizioni controllate di bilancio energetico positivo (introito calorico maggiore del dispendio energetico) o negativo (introito calorico minore del dispendio energetico), portando all'identificazione di due diversi fenotipi [18]: 1) "risparmiatore", un fenotipo ad alta efficienza energetica caratterizzato da una maggiore diminuzione di EE durante il digiuno e minor aumento di EE durante la sovralimentazione (200\% del fabbisogno energetico); e 2) "dissipatore", un fenotipo con minor diminuzione di EE durante il digiuno e maggior aumento di EE durante la sovralimentazione. Inoltre, i soggetti "risparmiatori" (quelli con una maggiore diminuzione dell'EE durante il digiuno) aumentavano il peso corporeo dopo un periodo di followup di 6 mesi [18]. In uno studio ulteriore condotto in 12 soggetti con obesità sottoposti a 6 settimane di restrizione calorica, gli individui "risparmiatori" mostravano una perdita di peso inferiore rispetto ai soggetti "dissipatori" [19]. A ulteriore conferma, recentemente, è stato dimostrato che gli individui "risparmiatori" presentano un aumento maggiore del peso corporeo e della massa grassa dopo 6 settimane di sovralimentazione, rispetto ai soggetti "dissipatori" [20].

\section{Conclusioni}

La spesa energetica agisce come regolatore dell'assunzione di cibo attraverso un "sensore di energia" che consente di adeguare l'apporto energetico alle necessità imposte dalle variazioni dell'ambiente. A sua volta, l'apporto alimentare influenza consensualmente la spesa energetica attraverso meccanismi ancora non del tutto conosciuti. La risposta adattativa della spesa energetica a manipolazioni acute dell' apporto energetico (es. digiuno o sovralimentazione) ha permesso di identificare due distinti fenotipi metabolici: un fenotipo "risparmiatore" di energia, più incline allo sviluppo dell'obesità e un fenotipo "dissipatore" di energia, più resistente all'aumento del peso corporeo. Una migliore comprensione delle complesse interazioni tra dispendio energetico e introito calorico dovrebbe facilitare lo sviluppo di interventi terapeutici e preventivi atti a contrastare la diffusione dell'obesità.

Informazioni Supplementari La versione online contiene materiale supplementare disponibile su https://doi.org/10.1007/s40619-02101007-y.

\section{Dichiarazioni etiche}

Conflitto di interesse Gli autori Alessio Basolo, Paola Fierabracci e Ferruccio Santini dichiarano di non avere conflitti di interesse.

Consenso informato Lo studio presentato in questo articolo non ha richiesto sperimentazione umana.

Studi sugli animali Gli autori di questo articolo non hanno eseguito studi sugli animali.

Open Access This article is licensed under a Creative Commons Attribution 4.0 International License, which permits use, sharing, adaptation, distribution and reproduction in any medium or format, as long as you give appropriate credit to the original author(s) and the source, provide a link to the Creative Commons licence, and indicate if changes were made. The images or other third party material in this article are included in the article's Creative Commons licence, unless indicated otherwise in a credit line to the material. If material is not included in the article's Creative Commons licence and your intended use is not permitted by statutory regulation or exceeds the permitted use, you will need to obtain permission directly from the copyright holder. To view a copy of this licence, visit http://creativecommons.org/licenses/by/4.0/.

\section{Bibliografia}

1. World Health Organization (2016) Obesity and overweight fact sheet. https://www.who.int/news-room/fact-sheets/detail/obesityand-overweight. Accessed on 18 September 2021

2. Ravussin E, Lillioja S, Anderson TE et al (1986) Determinants of 24-hour energy expenditure in man. Methods and results using a respiratory chamber. J Clin Invest 78(6):1568-1578

3. Morton GJ, Cummings DE, Baskin DG et al (2006) Central nervous system control of food intake and body weight. Nature 443(7109):289-295

4. Lam YY, Ravussin E (2016) Analysis of energy metabolism in humans: a review of methodologies. Mol Metab 5(11):1057-1071

5. Lam YY, Ravussin E (2017) Indirect calorimetry: an indispensable tool to understand and predict obesity. Eur J Clin Nutr 71(3):318-322

6. Piaggi P, Thearle MS, Bogardus C, Krakoff J (2013) Lower energy expenditure predicts long-term increases in weight and fat mass. $\mathbf{J}$ Clin Endocrinol Metab 98(4):E703-707

7. Ravussin E, Bogardus C (1992) A brief overview of human energy metabolism and its relationship to essential obesity. Am J Clin Nutr 55(1 Suppl):242s-245s

8. Bogardus C, Lillioja S, Ravussin E et al (1986) Familial dependence of the resting metabolic rate. N Engl J Med 315(2): 96-100 
9. Levine JA (2004) Non-exercise activity thermogenesis (NEAT). Nutr Rev 62(7 Pt 2):S82-S97

10. Thearle MS, Pannacciulli N, Bonfiglio S et al (2013) Extent and determinants of thermogenic responses to 24 hours of fasting, energy balance, and five different overfeeding diets in humans. J Clin Endocrinol Metab 98(7):2791-2799

11. Acheson KJ, Ravussin E, Wahren J, Jéquier E (1984) Thermic effect of glucose in man. Obligatory and facultative thermogenesis. J Clin Invest 74(5):1572-1580

12. Segal KR, Gutin B, Nyman AM, Pi-Sunyer FX (1985) Thermic effect of food at rest, during exercise, and after exercise in lean and obese men of similar body weight. J Clin Invest 76(3):1107-1112

13. Weise CM, Hohenadel MG, Krakoff J, Votruba SB (2014) Body composition and energy expenditure predict ad-libitum food and macronutrient intake in humans. Int J Obes, Lond 38(2):243-251

14. Ravussin E, Lillioja S, Knowler WC et al (1988) Reduced rate of energy expenditure as a risk factor for body-weight gain. N Engl J Med 318(8):467-472

15. Luke A, Durazo-Arvizu R, Cao G et al (2006) Positive association between resting energy expenditure and weight gain in a lean adult population. Am J Clin Nutr 83(5):1076-1081

16. Basolo A, Votruba SB, Heinitz S et al (2018) Deviations in energy sensing predict long-term weight change in overweight native Americans. Metabolism 82:65-71
17. Weyer C, Vozarova B, Ravussin E, Tataranni PA (2001) Changes in energy metabolism in response to $48 \mathrm{~h}$ of overfeeding and fasting in Caucasians and Pima Indians. Int J Obes Relat Metab Disord 25(5):593-600

18. Schlögl M, Piaggi P, Pannacciuli N et al (2015) Energy expenditure responses to fasting and overfeeding identify phenotypes associated with weight change. Diabetes 64(11):3680-3689

19. Reinhardt M, Thearle MS, Ibrahim M et al (2015) A human thrifty phenotype associated with less weight loss during caloric restriction. Diabetes 64(8):2859-2867

20. Hollstein T, Ando T, Basolo A et al (2019) Metabolic response to fasting predicts weight gain during low-protein overfeeding in lean men: further evidence for spendthrift and thrifty metabolic phenotypes. Am J Clin Nutr 110(3):593-604

Nota della casa editrice Springer Nature rimane neutrale in riguardo alle rivendicazioni giurisdizionali nelle mappe pubblicate e nelle affiliazioni istituzionali. 